Revue d'histoire de l'enfance « irrégulière »

Le Temps de l'histoire

$11 \mid 2009$

Paroles libres, paroles captives

\title{
Petits maraudeurs et jeunes vauriens puydomois.
}

Représentation médiatique des accusés mineurs jugés par la cour d'assises du Puy-de-Dôme du Second Empire à la Belle Epoque

(1852-1912.

Sébastien Soulier

\section{(2) OpenEdition}

Journals

Édition électronique

URL : http://journals.openedition.org/rhei/3092

DOI : 10.4000/rhei.3092

ISBN : 978-2-7535-1650-2

ISSN : 1777-540X

Éditeur

Presses universitaires de Rennes

Édition imprimée

Date de publication : 1 octobre 2009

ISBN : 978-2-7535-0927-6

ISSN : 1287-2431

Référence électronique

Sébastien Soulier, "Petits maraudeurs et jeunes vauriens puydomois. », Revue d'histoire de l'enfance " irrégulière » [En ligne], 11 | 2009, mis en ligne le 01 octobre 2009, consulté le 10 décembre 2020. URL http://journals.openedition.org/rhei/3092 ; DOI : https://doi.org/10.4000/rhei.3092

Ce document a été généré automatiquement le 10 décembre 2020.

(C) PUR 


\title{
Petits maraudeurs et jeunes vauriens puydomois.
}

\author{
Représentation médiatique des accusés mineurs jugés par la cour \\ d'assises du Puy-de-Dôme du Second Empire à la Belle Epoque \\ (1852-1912.
}

\section{Sébastien Soulier}

1 De 1852 à 1912, la cour d'assises du Puy-de-Dôme juge 51 accusés âgés de 12 à 16 ans auxquels il faut ajouter, à partir d'août 1906, 9 accusés de 17 et 18 ans considérés comme mineurs pénaux par la loi du 12 avril $^{1}$. Avec une moyenne d'un accusé par an, il s'agit donc d'une criminalité d'exception. Qui plus est, la loi stipulant que seuls les crimes les plus graves commis par des mineurs sans complices doivent être jugés par la cour d'assises ${ }^{2}, 70 \%$ des accusés présents sur les bancs du Palais de Justice ont agi accompagnés d'un ou plusieurs individus plus âgés. Si la population concernée par ces affaires appartient en grande partie au monde rural (63,3\% des accusés), on observe également une représentation assez importante de jeunes évoluant dans les milieux industrialisés ou semi-industrialisés de Clermont-Ferrand, d'Issoire et de Thiers. Evitons toutefois une division trop simpliste entre deux jeunesses qui s'opposeraient, l'une des campagnes et l'autre des villes. Les passerelles géographiques, sociales et professionnelles entre ces deux mondes sont multiples et complexes. Encore en 1912, la forte ruralité qui caractérise la région laisse une empreinte indélébile sur le territoire dit « urbain ». Essentiellement masculine (87 \% des accusés), la majorité des crimes commis sont des cambriolages (51 \% des accusés). Les homicides volontaires, infanticides compris, représentent $10 \%$ des accusés, suivis des crimes sexuels $(8,3 \%)$, des vols avec violence et des incendies volontaires $(6,7 \% \text { chacun })^{3}$. Le mineur criminel que l'on rencontre le plus souvent est donc un jeune complice cambrioleur âgé de 15 ou 16 ans, d'origine rurale mais résidant souvent en ville. L'exposition médiatique des crimes commis par des mineurs provoque-t-elle une réaction au sein de l'opinion publique, une inquiétude que la presse entretiendrait à travers un discours alarmiste sur l'éducation de la jeunesse et la décadence des mœurs? Comme sur le reste du territoire national, le développement de la presse puydomoise est en grande partie lié aux évolutions du contexte politique, aux initiatives personnelles et aux 
moyens matériels et financiers des gérants et des imprimeurs locaux. Sous le Second Empire, deux principaux journaux gouvernementaux se disputent le lectorat clermontois : l'Ami de la Patrie, remplacé par le Moniteur du Puy-de-Dôme en 1856, et le Journal du Puy-de-Dôme de 1852 à 1860. L'avènement de la IIIe République et la loi de 1881 s'accompagnent d'une multiplication et d'une diversification des feuilles politiques départementales, le désormais républicain Moniteur du Puy-de-Dôme, devenu le plus important quotidien du département, doit alors composer avec une presse radicale (le Petit Clermontois de 1885 à 1894) et une presse catholique conservatrice (la Gazette d'Auvergne de 1872 à 1888, la Dépêche du Puy-de-Dôme de 1889 à 1896 et l'Avenir du Puy-de-Dôme à partir de 1896). Cette période, marquée à partir des années 1890 par la naissance et le développement d'une presse populaire plaçant l'actualité sensationnelle au centre de sa stratégie commerciale, transformera en profondeur la forme et le contenu des journaux puydomois : le règne du fait divers ne fait que commencer. Le débat politique sur l'avenir de ces jeunes et sur la réponse à donner à leurs déviances at-il lieu dans les colonnes de ces quotidiens et hebdomadaires départementaux ? A travers les différents représentants de la presse puydomoise, nous tenterons de répondre à ces questions et d'exposer la représentation médiatique d'un phénomène de société qui bénéficiera, à la veille de la Grande Guerre, d'un engouement médiatique d'exception à travers la figure de l'Apache parisien ${ }^{4}$. Comme point de départ de notre réflexion, nous interrogerons deux aspects du regard porté par la chronique judiciaire sur les mineurs criminels : l'appréciation de leur appartenance communautaire et la construction des portraits physiques et comportementaux de ces enfants pendant les débats de la cour d'assises. Nous poursuivrons nos recherches en portant notre attention sur l'exposition des facteurs criminogènes et des comportements transgressifs associés à la criminalité juvénile. Nous en distinguerons trois : la précarité familiale, l'errance sociale et la perversité. Enfin, parce qu'elle est indissociable de toute étude des phénomènes criminels, la réponse judiciaire apportée à la délinquance des mineurs sera au centre de notre troisième partie. Nous en étudierons, ici aussi, son écho médiatique à travers deux thèmes distincts : la défense et la sanction.

\section{Le regard de la presse}

\section{Quelle jeunesse? Appréciation de l'appartenance communautaire des jeunes accusés.}

2 En abordant ces manifestations locales de la criminalité juvénile, nous pensions trouver un vif débat, à la fois politique et médiatique, sur le devenir de la population et sur les risques du développement de ces déviances. Un débat qui s'accompagnerait irrémédiablement d'une stigmatisation de l'idéologie politique adverse et d'un avertissement vis-à-vis du déclin des valeurs morales, notamment à partir des années 1880. Rien de cela n'apparaît dans les articles que nous avons parcourus. Il aurait sans doute fallu, pour qu'une réflexion de ce type naisse et qu'un débat s'anime, que ces jeunes criminels soient considérés comme intégrés dans le tissu social des communautés urbaines et rurales concernées, qu'ils représentent symboliquement l'ensemble de la jeunesse sur laquelle planerait le néfaste présage d'une dissolution des mœurs ou d'un engagement idéologique corrupteur. Or ce n'est pas le cas : la presse distingue clairement ces jeunes déviants du reste de la population. Marginalisés, on les incorpore au sein d'une catégorie de jeunes malfaiteurs qui bien souvent n'ont d'autre 
destinée que celle vouée au crime. «En refusant de se reconnaître à travers eux, la société s'interroge sur leur degré d'humanité et ne veut voir en eux que des individus s'étant toujours inscrits hors des normes et porteurs des stigmates de la monstruosité ", nous rappelle Sylvie Lapalus à propos de la perception des jeunes parricides ${ }^{5}$ en de nombreux points identique à celle des mineurs que nous étudions ici. Si de surcroît l'enfant est étranger à la localité ou au pays, le processus d'exclusion de l'élément déviant est encore plus net. Quand on découvre l'infanticide commis par Anne Miallet le 21 juillet 1876 à Joze, on rappelle que la jeune domestique n'est pas originaire de cette localité : «l'honneur des jeunes Jozelères reste immaculé » ajoute le Moniteur du Puy-de-Dôme $e^{6}$. Et pourtant, ce sont bel et bien les enfants du pays que l'on retrouve en majorité sur le banc des accusés : $70 \%$ des mineurs jugés vivent dans leur commune d'origine ou à proximité et plus de $75 \%$ d'entre eux commettent leur crime dans la commune où ils sont domiciliés. Difficile toutefois de déceler, dans les actes perpétrés par ces mineurs, le quelconque reflet d'un sentiment d'appartenance à une communauté villageoise comme l'a observé Jean-Claude Farcy dans son étude des rixes villageoises ${ }^{7}$, excepté dans un cas particulier: le crime de Cournon, où huit jeunes habitants de cette localité sont jugés le 11 février 1874 pour avoir frappé à mort, en pleine rue, un ouvrier étranger à la commune. La Gazette d'Auvergne rappelle que « cette journée décidera du sort de ces huit jeunes gens, qui vont pour la plupart échanger les jours de travail en plein soleil, au milieu des champs, contre les travaux des forçats et des réclusionnaires $»^{8}$. Le quotidien monarchiste reconnaît ici l'appartenance des accusés à la population cournonaise, tout en insistant sur les mœurs archaïques du milieu rural : «Il existe, paraît-il, à Cournon, parmi les jeunes indigènes, un usage, passablement inhospitalier, qui consiste à précipiter dans le bac d'une fontaine les ouvriers étrangers à la localité. On appelle cela: faire subir le bardeau.. ${ }^{9}$ Si nous retrouvons ici la traditionnelle critique par la notabilité urbaine de la violence du monde rural, la stigmatisation par la presse de la jeunesse paysanne reste toutefois exceptionnelle dans les affaires de crimes commis par des mineurs, et la comparaison entre deux jeunesses, une des villes et l'autre des campagnes, n'est pas systématique dans la rhétorique du chroniqueur judiciaire. Qui plus est, l'affaire de Cournon dépasse le cadre de notre réflexion: un seul des accusés, Michel Dhermain, est âgé de moins de 17 ans et le rôle qu'il tient dans le spectacle judiciaire se résume à une simple présence, une figuration presque invisible. On ne s'attarde ni sur lui, ni sur son interrogatoire, ni sur son verdict.

Purgés de toute considération politique ou idéologique, les jugements de la presse à l'égard de ces mineurs sur lesquels aucun symbole ni enjeu social ne pèse se résument finalement à une indignation sommaire, quelques regrets que l'on affiche face à ces destins brisés qui n'intéressent plus personne : "C'est un spectacle affligeant que de voir conduire sur le banc, nous ne dirons pas trois jeunes gens, mais trois enfants, pour répondre à une accusation très grave, celle du vol d'une somme de 580 francs ", lit-on en introduction du compte rendu d'audience de l'affaire Bas, Sureau et Chassignol en décembre $1855^{10}$. Plus l'accusé est jeune, plus la scène est navrante : «En entrant dans la salle, au premier regard que l'on dirige sur le banc des accusés, on se sent le cœur péniblement serré. On y cherche un incendiaire habile et redoutable (...) et on y trouve, pour répondre à cette énorme accusation, un tout petit garçon dont la tête à peine dépasse le dossier du banc de la défense ", relève le Moniteur du Puy-de-Dôme ${ }^{11}$ lors du procès du jeune André Mondière, accusé de quatre incendies en moins de quinze jours dans la commune de Saint-Joannis. Le ton ne change pas une trentaine d'années plus 
tard lorsque l'on présente le jeune Joseph Prat, âgé de 12 ans, accusé d'avoir incendié une école communale dirigée par les frères de la congrégation du Sacré-Cœur à Chaumont : "Un criminel de 12 ans qui comparaît devant le jury, n'est-ce pas triste ! Profondément triste! $»^{12}$. Du gâchis, c'est ainsi que nous pourrions résumer le sentiment qui imprègne les lignes de la chronique quand elle relate ce type d'affaires et quand elle présente cette génération d'accusés. Du milieu du XIXe siècle à la veille de la Grande Guerre, la perception de cette jeunesse perdue par la chronique n'évolue guère, l'existence de ces enfants se livre aux yeux du lectorat sous un mauvais jour, et un seul: une fois condamnés, ils n'existent plus.

\section{Portrait physique et interprétation des attitudes.}

4 La description physique et l'interprétation des attitudes des mineurs assis sur le banc de la salle d'audience n'obéissent pas aux règles traditionnelles du portrait médiatique de criminel que l'on pourrait résumer de la façon suivante : déceler le mal coûte que coûte dans les traits du visage, dans la profondeur du regard, dans la gestuelle et la parole d'un accusé. En effet, dans la majorité des cas, les descriptions physiques sont plutôt favorables aux accusés. Pour ne citer que deux exemples, Françoise Thévenet, accusée de vol domestique en mai 1853, apparaît comme une jeune fille "fraîche et jolie $»^{13}$ et le jeune Annet Roussel, complice de son père dans une affaire d'homicide volontaire, "est doué d'une physionomie aimable où brillent deux grands yeux intelligents $»^{14}$. Le regard, élément central où se décèle l'empreinte du mal n'a ici rien d'étrange. L'acte criminel ne semble pas avoir enlaidi la beauté de ces enfants, et quand le physique est disgracieux, on l'associe rarement à une tare morale. "Sans être douce ", la physionomie de Jacques-Louis Ventalon, jugé pour meurtre le 27 mai 1871 " semble démentir la grave accusation qui pèse sur lui ». ${ }^{15} \mathrm{Et}$ pourtant, « malgré leurs airs débonnaires (...), ce sont d'audacieux et dangereux voleurs » avertit Le Moniteur du Puy-de-Dôme ${ }^{16}$ en observant Jules Chauvon âgé de 16 ans et son complice Jean Charguellon, accusés de vols qualifiés en mai 1887. La description physique des accusés obéit à une toute autre intention que la recherche d'une matérialisation physique du mal. Leur apparence constitue en effet un élément incontournable dans l'appréciation de leur statut d'enfant. Antoine Champommier, accusé de viol en 1853, est âgé de 16 ans et 3 mois mais «n'en paraît pas $12 »^{17}$, « quel crime peut avoir commis cet enfant? se disaient les nombreux spectateurs qui encombraient la salle d'audience. $\aleph^{18}$ Le jeune Antoine n'est d'ailleurs pas le seul à paraître plus jeune sous la plume des chroniqueurs : Jean Bas et Auguste Surot sont « des accusés de 14 à 15 ans (...) qui en paraissent 12 à peine $»^{19}$; le jeune Bourguignon, jugé pour vol avec violence, est âgé de 16 ans mais « en parait $12 »$ aussi $^{20}$. Enfin Joseph Boudol, accusé de vol qualifié, également dans sa seizième année, " a l'extérieur d'un gamin de 10 à 12 ans ». ${ }^{21}$ Un rajeunissement caricatural des accusés que l'on retrouve également dans la Gazette des Tribunaux quand elle insiste "dans ses titres sur la jeunesse de ces accusés un peu particuliers, les rajeunissant au besoin dans la description qu'elle en fait $\$ . .^{22}$ La douzième année apparaît donc comme l'achèvement d'une première enfance : Jeanne Bard, accusée d'incendie volontaire en novembre 1859 et envoyée en maison de correction, est «à peine sortie de l'enfance ", elle "vient seulement d'atteindre sa quatorzième année ». ${ }^{23}$ Reste à découvrir si cette limite répond à des perceptions socioculturelles de l'enfance ou si, plus simplement, elle n'est que l'écho des limites définies par le code pénal pour invoquer l'absence de discernement. Jean-Claude Vimont nous rappelle qu'« au-delà 
des normes juridiques qui définissent les mineurs de justice (...), des catégorisations issues de représentations accompagnent les jeunes délinquants et ce sont elles, bien plus sûrement que le droit, qui ont déterminé le mode de répression judiciaire ${ }^{24} \mathrm{et}$, ajouterons-nous, qui déterminent la perception de ces jeunes par la chronique judiciaire. L'étude du vocabulaire utilisé dans les comptes rendus d'audiences renvoie à la même réalité des perceptions générationnelles. On associe très largement les mots enfant et petit aux accusés de moins de 14 ans (misérable enfant, petit vagabond, petit maraudeur etc.) alors que les accusés de 15 à 16 ans seront davantage désignés comme de jeunes garçons ou de jeunes hommes. Notons également l'évocation suggérée du passage de l'enfance à l'adolescence à travers l'emploi d'expressions telles que presque enfant ou presque gamin pour des accusés âgés de seize ans. Ici aussi, difficile de distinguer clairement, dans la chronique, une évolution dans la perception des différentes strates de la jeunesse au fil des décennies qui séparent les premières années du règne de Napoléon III aux dernières années de la Belle Epoque. En ce qui concerne les jeunes filles, la palette des désignations est bien moins fournie : l'emploi de jeune fille et de fille (suivi du nom de famille) sont les expressions les plus souvent rencontrées. On peut toutefois, dès l'âge de 15 ans, être désignée comme femme.

5 Au-delà des attributs physiques, le comportement des accusés pendant l'instruction et au cours du procès influencent inévitablement la perception que l'on a d'eux. Les sanglots demeurent, à ce propos, la manifestation émotionnelle la plus à même de rappeler qu'avant d'être un criminel, l'accusé reste un enfant. La pression et la solennité de la justice ne laissent pas indifférent, et chaque étape d'un procès est propice aux crises de larmes. Le jeune Riffard, clerc d'avoué accusé d'avoir volé son patron, subit «la tête baissée et au milieu des larmes » l'interrogatoire du président Cantillion ${ }^{25}$. Mais c'est bien l'audition du verdict et les quelques minutes précédant le retour des jurés qui constituent le point culminant du drame : chacun des membres de la bande de Cournon « redoute le résultat de ces solennels débats et ceux-là mêmes qui conservent encore quelque espoir ont le cœur serré par l'angoisse $»^{26}$. Quant au jeune Champommier, il «fondait en larmes $»^{27}$ en apprenant que la justice retenait contre lui le crime d'attentat à la pudeur et que, de ce fait, il réintégrait la maison d'arrêt. L'insouciance ${ }^{28}$ de certains accusés peut également être perçue comme une marque de comportement infantile. Parfois associée à de la perversité, nous y reviendrons, l'attitude désinvolte des plus jeunes effraie ou amuse, selon les circonstances et surtout selon le capital de sympathie de l'accusé auprès de l'auditoire. «On frémit quelque peu en voyant le sang-froid de ces deux jeunes scélérats racontant l'un et l'autre leur crime avec une insouciance qui soulève souvent les murmures de l'auditoire ", nous dit le Petit Clermontois $^{29}$ en évoquant les comportements de Jean-Baptiste Binet et Jean-Baptiste Convert, accusés de vol commis avec violence. A l'inverse, les réponses naïves du jeune Lukasienviez, jugé pour un cambriolage commis à Clermont-Ferrand dans la nuit du 19 au 20 mai 1890, provoquent dans l'auditoire "une douce hilarité $»^{30}$, alors que l'assurance des frères Chassagnon, également accusés de vols qualifiés, «excite des sourires $~^{31}$. L'agressivité est un trait de caractère rarement rencontré et ce, malgré la réputation parfois sulfureuse de quelques-uns des accusés. Jacques-Louis Ventalon " passait avec raison pour être d'un caractère violent et brutal »" , ce qui ne l'empêche pas, pendant le procès, d'éclater en sanglots en affirmant ne pas avoir tué sa victime dans le but de la voler. ${ }^{33}$ Quand cette agressivité apparait toutefois, sporadiquement, l'accusé retrouve très rapidement un statut de dangereux criminel, et la figure du mal émerge là où on ne l'attendait plus. Annet Roussel avait, rappelons-nous, une attitude 
«des plus correctes » au début de son procès. C'est un enfant qui répond «avec beaucoup de calme aux questions qui lui sont posées $»^{34}$. Mais quand le témoin Dufraisse affirme catégoriquement l'avoir vu avec son père près du lieu du crime, le jeune homme s'emporte: «il se lève, brusquement, comme mû par un ressort et s'écrie : «Dufraisse ment. J'ai connu son frère à la maison d'arrêt, qui m'a dit qu'il était capable de commettre le crime. » Des murmures s'élèvent. Les accusés qui, jusque là, n'étaient pas antipathiques, le deviennent maintenant par leur attitude $» .^{35}$

\section{Facteurs criminogènes et comportements transgressifs}

\section{Familles précaires : de la défaillance éducative à la complicité criminelle.}

6 La précarité des conditions de vie dans les familles d'où sont issus $38,3 \%$ des jeunes accusés ${ }^{36}$ constitue régulièrement aux yeux de la justice et de la chronique un facteur criminogène évident : on associe volontiers misère sociale et dépravation des mœurs, et par extension penchant pour le crime. La misère de la famille d'Anne Chelle, accusée de vol domestique, "étant de notoriété publique, les soupçons prirent une certaine gravité », lit-on dans Le Moniteur du Puy-de-Dôme. ${ }^{37}$ Dans les faits et pour l'enfant, cette misère se concrétise le plus souvent par des conditions de vie difficile, une absence de suivi scolaire, l'absence d'un des deux parents, l'alcoolisme, la violence conjugale, une éducation brutale, etc. Quand le président questionne le jeune Joseph Prat sur son éducation, il répond que sa mère le «fichait, lisez me battait ", et que c'est pour cela qu'il s'était "envolé ${ }^{38}{ }^{38}$ François Aussize, âgé de 12 ans, est accusé de coups et blessures mortels sur son père en 1871 : « fils d'un forçat libéré et d'une mère de mœurs décriées, [il] a contracté au milieu des scandales quotidiens de la maison paternelle, des habitudes précoces d'indiscipline et de violence ». Voilà qui résume parfaitement le sentiment largement palpable dans les discours judiciaire et médiatique à propos de cette jeunesse considérée comme perdue. Il voulait protéger sa mère des coups répétés d'un mari brutal, «mais si légitime que soit (...) le sentiment qui a armé la main du jeune Aussize, il ne saurait l'absoudre entièrement de l'acte qu'il a commis ». La victimisation des enfants évoluant dans les cercles sociaux difficiles se lit entre les lignes, mais elle ne constitue en rien une circonstance atténuante quand le crime se révèle brutal et sanglant. Les parents complices, poussant leur enfant à commettre un crime ou y participant activement représente $11,7 \%$ des affaires mettant en scène des accusés mineurs. Cette participation des parents concerne principalement des vols domestiques et les cambriolages. Si Françoise Thévenet s'est rendue coupable d'un vol chez sa patronne, c'est qu'elle n'a agi que "par les instigations de sa mère (...), cette femme dont les conseils perfides ont cherché à perdre son enfant qui n'a pas atteint sa seizième année ", lit-on dans Le Journal du Puy-de-Dôme. ${ }^{39}$ Quand on juge la famille Roubille, le 18 août 1880 , pour le cambriolage de la maison voisine de la leur, on apprend que le jeune Félix, 15 ans, et ses deux sœurs n'ont fait que suivre les instructions de leur mère. ${ }^{40}$ Les mères de famille ne sont pas les seules à inciter leurs enfants à commettre des vols et à participer plus ou moins activement aux actes de leurs enfants. Si Jean Chauvon persiste à dire qu'il n'a pas « excité Charguellon et son fils » à commettre le vol des troncs de l'église de Ronzières dans la nuit du 24 au 25 
janvier 1887, selon le Moniteur du Puy-de-Dôme, le doute n'est pas permis : c'est bel et bien le père qui est «l'âme de ce complot »"

7 Les affaires d'homicide volontaire mettant en cause une association parent-enfant laissent apparaître un tout autre degré de culpabilité du mineur à l'égard des actes commis. L'incitation au crime par l'autorité parentale n'est plus aussi évidente que dans les affaires précédentes, et l'accusé perd son statut de victime influencée pour prendre celui de complice à part entière. La question de la responsabilité de Mathilde Touche, accusée d'assassinat, de vols qualifiés et de tentative d'empoisonnement avec la complicité de sa mère Marie Pradel en août 1903 à Culhat, suscite un certain nombre de questions : « on s'est demandé si elle n'agissait pas sous une influence maladive. Des médecins ont été chargés de l'examiner, ils l'ont reconnue robuste $\$^{42}$ Qui plus est, Mathilde Touche est une fille-mère et fréquente plusieurs amants, ce qui achève de lui conférer un statut de jeune adulte aux mœurs dissolues qui finalement n'a plus rien d'une enfant. Dans l'affaire Roussel, un casseur de pierre de Sayat est accusé d'avoir assassiné un compagnon de travail avec la complicité de son fils de 15 ans sur un chemin public dans la nuit du 23 au 24 mars 1907. Plus que le fils, c'est la famille que l'on craint et qui est montrée du doigt : ils ont « une très mauvaise réputation, ils sont violents et redoutés de la population. La rumeur publique leur attribue d'autres méfaits restés impunis : ils inspirent à leurs voisins une sorte de terreur $\gg{ }^{43} \mathrm{Si}$ l'association père-fils génère une animosité perceptible dans les murmures de la salle d'audience, le jeune âge d'Annet Roussel n'attire aucunement l'attention de la chronique et des spectateurs : «Pendant la suspension d'audience, le public se montre extrêmement bruyant. On discute les dépositions des témoins, les explications des accusés, les uns condamnent à l'avance, les autres acquittent avant que le jury ait rendu son verdict $» .^{44}$

\section{L'errance criminelle : la paresse, l'alcool et la débauche.}

8 L'absence d'activité professionnelle, la fréquentation des cabarets et des maisons de tolérance, autrement dit la paresse, l'alcool et la débauche, sont des éléments que l'on retrouve dans une très grande majorité des affaires mettant en scène des mineurs. S'il ne s'agit pas de déviances exclusivement associées aux mineurs, le rappel de l'absence d'une activité professionnelle et d'un mépris chronique du travail doit être compris ici comme le signe évident d'un refus d'intégration aux communautés auxquelles ces jeunes appartiennent, le rejet d'une norme qui veut que chacun obtienne sa place dans la société en fonction de ce qu'il peut y apporter par son labeur. Guillaume-Jules Cerciron, jugé pour vols qualifiés le 13 novembre 1871, fait partie d'une bande dont les membres « ne se livrent habituellement à aucun travail ». ${ }^{45}$ Lukasienviez, " fumiste de son état, est paresseux et doué d'autres qualités aussi recommandables $\${ }^{46}$ Dans le canton de la Chaise-Dieu, les frères Chassagnon, accusés de vol, ont également «la réputation d'être paresseux " dans la commune de Novacelles. ${ }^{47}$ A cette absence de toute volonté de travailler s'ajoute une attirance précoce pour la débauche et l'alcool : "les accusés sont mal famés, ils ne travaillent pas, frayent les cabarets, s'adonnent à l'ivrognerie et passent souvent les nuits hors de chez eux » lit-on dans le Moniteur du Puy-de-Dôme à propos de Gouay et son complice, jugés pour trois cambriolages en février $1882 .{ }^{48}$ Une fois leur forfait commis, Chassignol, Bas et Sureau « se partagèrent (...) la somme volée, et l'employèrent en orgie dans les cabarets et les mauvais lieux ». ${ }^{49}$ 
Le butin de l'agression commise par Antoine Bon et Jean Mandaroux dans la soirée du 16 octobre 1870 fut également "promptement dissipé par eux dans des maisons publiques $\aleph^{50}$. Cette apparente futilité du mobile des vols, boire et fréquenter des prostituées, s'expose largement dans les colonnes des quotidiens, entretenant l'indignation du lectorat à l'égard d'une population inutile qui, en plus de ne pas travailler, souhaite accéder à des besoins primaires et immoraux, des besoins que l'absence de moyens de subsistance ou la faiblesse des salaires ne permettent pas d'assouvir quotidiennement. Dans l'affaire des soustractions commises en juin 1865 par les employés Fioux, Peschaud et Debreuil-Monnet sur leur lieu de travail, les marchandises volées avaient été portées dans la maison de prostitution tenue par le nommé Gagnardre, rue des Trois-Raisins, $\mathrm{N}^{\circ} 16$. «Leurs faibles salaires ne pouvaient suffire à leurs dépenses, et, suivant l'accusation, ils obéirent bientôt aux suggestions des filles publiques de cette maison en volant chez leur patron des objets de toilette ", apprend-on dans Le Moniteur du Puy-de-Dôme..$^{51}$

9 Le comportement anormal et transgressif de ces mineurs aboutit, inévitablement, à une marginalisation de l'individu qui, quand il pénètre dans la salle d'audience, trâne derrière lui la plus mauvaise des réputations. Sur les 60 mineurs jugés par la cour d'assises du Puy-de-Dôme, 38 ont, selon l'instruction et/ou la presse, une mauvaise réputation dans le pays dont ils sont originaires ou domiciliés. Une mauvaise réputation qu'ils doivent également à leur vagabondage intempestif et aux multiples larcins impunis dont ils sont suspectés par les populations et les autorités locales. Si nous trouvons peu d'enfants sans domicile fixe ou vivant loin de leur pays d'origine, un grand nombre d'entre eux vagabondent au sein de leur localité. Jean-Jacques Yvorel rappelle, dans sa réflexion sur les enfants vagabonds, combien la notion même de vagabondage mérite une attention toute particulière. ${ }^{52}$ Sorties nocturnes, maraudage, filouterie, école buissonnière, mendicité, ce vagabondage, entendons par là cette transgression du quotidien normalisé des jeunes de leur génération et de leur localité, occupe une place importante dans la retranscription des débats. Et si le parallèle entre vagabondage et comportement criminel n'est pas explicitement dénoncé, il semble admis par tous : un enfant qui vagabonde est un enfant potentiellement criminel. Le jeune Queyriaux, accusé de vol qualifié à 16 ans, « appartient à une honnête famille de cultivateurs. Mais (...) il commence à se faire remarquer par sa mauvaise conduite, jouant aux cartes sur la place de Jaude et rôdeur nocturne aux allures suspectes $»{ }^{53}$ Quant au jeune Prat, son père le forçait quotidiennement à aller à l'école : «mais je n'y allais pas », répond fièrement l'enfant de 12 ans, «j'étais un mauvais sujet ! ${ }^{54}$

\section{La perversité qui mène au crime.}

10 Si la précarité des situations familiales et les comportements transgressifs sont régulièrement évoqués dans le récit des crimes des jeunes accusés, l'autre facteur criminogène incontournable aux yeux de la chronique judiciaire est sans aucun doute l'existence d'une perversité précoce, innée, inexplicable et incurable. Une perversité qui fait glisser inexorablement l'enfant vers un point de non-retour. Cette idée d'un mauvais chemin pris par l'enfant, d'une ascension vers le crime dont l'ultime étape reste bien évidemment l'homicide volontaire, est évoquée à plusieurs reprises dans les dossiers de procédure criminelle et dans les colonnes des journaux. «Les accusés ont à peine franchi le seuil de la vie que déjà ils sont engagés dans le sentier large et glissant du crime; et ils s'y enfoncent en aveugles jusqu'à ce que le terrain mouvant qu'ils 
foulent se dérobe sous leurs pas ", lit-on dans l'Ami de la Patrie à propos de Simon Chassignol, Jean Bas et Pierre-Auguste Surot..$^{55}$ L'acte d'accusation de l'affaire Aimard nous apprend que les jeunes Perrier et Queyriaux «n'ont manifesté leurs mauvais instincts que dans ces derniers temps, mais ils semblent avoir fait de rapides progrès dans le mal $»{ }^{56}$ Quant aux jeunes Binet et Convert, « rien ne faisait supposer qu'ils auraient un début aussi odieux dans la voie du crime $»{ }^{57}$ Cette perversité se révèle essentiellement à travers le passé des jeunes accusés et d'après la façon dont ils ont perpétré leur crime: "Les faits graves relevés par l'information à la charge de Démarchi (...) dénotent chez leur auteur âgé de moins de 17 ans, une perversité précoce et une rare audace » affirme l'Indépendant $d u$ Centre. ${ }^{58} \mathrm{Le}$ substitut du procureur Féry d'Esclands, dans son réquisitoire du 7 août 1890, montre « quelle perversité particulière Lukasienviez a déployée tant pendant l'accomplissement du vol que devant les magistrats et la Cour.$^{59} \mathrm{La}$ cruauté envers les animaux constitue un signe particulièrement fort de cette perversité. "En septembre 1855, aidé de plusieurs mauvais garnements dont il était le chef, [André Mondière] pourchassa si longtemps de petits cochons appartenant à Blaise que ces animaux furent forcés. Il voulait en tuer un, une femme l'empêcha ", lit-on dans l'acte d'accusation. "Vous étiez un enfant cruel ", déclare le président de l'audience en évoquant l'épisode. ${ }^{60}$ Les brutalités commises sur des moutons par Pierre Thuel et Baptiste Gouay pendant leur cambriolage « dénotent chez leurs auteurs une perversité précoce et des instincts barbares» ${ }^{61}$ selon le Riom Journal. Sylvie Lapalus nous rappelle que «les actes de barbarie envers les animaux, immanquablement épinglés dans le passé des criminels, sont lus a posteriori comme annonciateurs de la criminalité, au moment même où s'abaisse le seuil de tolérance à la violence $»{ }^{62}$ Quand la perversité touche les jeunes filles, on y ajoute systématiquement une dimension sexuelle. La jeune Anne Miallet a une réputation «déplorable au point de vue des mœurs ", ${ }^{63}$ tandis que la jeune Mathilde Touche est "une jeune fille vicieuse ${ }^{64}$ Par ailleurs, une des spécificités de cette perversité est qu'elle ne prive en rien l'enfant de son intelligence. Et quand les deux s'associent, de l'intelligence nait la ruse et l'audace, et l'on assiste à l'émergence des plus redoutables criminels qui, quel que soit leur âge, doivent être exclus de la société. "Ces jeunes dépourvus de sens moral, sans éducation morale, aux penchants pervers, ne bénéficiaient d'aucune indulgence, surtout lorsqu'ils avaient commis des actes délictueux en récidive ou en bande $» .^{65}$ On ne s'attarde pas, finalement, sur les origines et les causes de cette perversité, on se contente dans la plupart des cas de l'observer et de s'effrayer de sa précocité. Sans doute trouve-t-on dans la dénonciation d'un vice obscur le raccourci idéal pour expliquer des déviances incomprises, ou que l'on ne souhaite pas comprendre. Nous l'avons déjà souligné : la jeunesse que l'on juge aux assises n'est pas assimilée au reste de la population, et cette perversité constitue véritablement un des marqueurs de cette distinction. Plus qu'une véritable observation d'un travers psychologique, cette notion de perversité apparait davantage comme un élément récursif du champ lexical de la délinquance juvénile.

\section{Le mineur, la justice et la chronique}

\section{Ceux que l'on entraîne et ceux qui ne discernent pas}

11 Les extraits de plaidoiries présents dans les comptes rendus d'audience révèlent à quel point la défense des mineurs jugés par la cour d'assises obéit à des règles immuables qui 
ne changent guère d'un siècle à l'autre. Un système de défense à base d'un rejet des responsabilités sur les plus âgés et sur l'autorité familiale quand celle-ci est mise en cause. Nous avons déjà évoqué plusieurs fois les cas de Geneviève Grand et sa fille Françoise Thévenet, jugées pour vols domestiques. L'avocat général Ancelot réclame " une grande indulgence pour Françoise Thévenet, que son âge protège, et qui ne paraît avoir agi que par les conseils et les obsessions de sa mère ». Il invite le jury « à frapper fortement cette femme dont les avis ont mis sa malheureuse enfant dans la fâcheuse position où elle se trouve aujourd'hui $"{ }^{66}$ Même chose pour Marie Vincent, 16 ans, également accusée de vols domestiques, acquittée et envoyée en maison de correction. Sa co-accusée Anne Dugnat, pour déterminer la jeune Marie à commettre ces vols, lui disait: "A Issoire, tous les domestiques volent leurs maîtres; fais comme eux et tu seras largement récompensée $"{ }^{67}$ Quand on juge Achille Vaisse, 15 ans, Louis Retrus, 16 ans, et Jean Jouberton, 17 ans, pour un cambriolage commis chez des liquoristes de Clermont-Ferrand en 1875, le défenseur d'Achille prend la parole: «Par quelle pente est arrivé à l'abîme où il se trouve aujourd'hui le malheureux Vaisse? La cause de cet errement fatal, on la trouve dans la connaissance qu'il a faite de Retrus et de Jouberton. La vérité est que sans Retrus, sans Jouberton, Vaisse serait encore dans sa famille $» .^{68}$ Dans l'affaire de cambriolage Batisse et Giron, le défenseur du jeune Jean Batisse, 15 ans, "démontre l'inconscience de cet enfant, qui a cédé, non sans répugnance, aux suggestions, à l'influence de Giron, et a ainsi prêté son concours à une action dont il ne pouvait comprendre la portée $»{ }^{69}$ Les plaidoiries se suivent et se ressemblent. Quand on juge l'agression commise par Bourguignon et Saint-Cyr, la défense appuie encore son argumentation sur « le jeune âge de son client et surtout sur l'influence prise sur celuici par Saint-Cyr $» .^{70}$ Les meneurs entrainant les plus jeunes sur la pente du crime ne sont pas nécessairement présents sur le banc des accusés, ni toujours clairement identifiés. Pour le jeune Chassagnon, ce sont les mauvais exemples (...) donnés pendant sa première jeunesse » qui, selon son avocat, sont à l'origine de ses déviances..$^{71}$ Quant au jeune Lukasienviez, le seul cambrioleur à avoir été attrapé dans l'affaire du vol de la Rue Neuve, il n'est, selon son défenseur, qu'un « complice inconscient $» .^{72}$

Pour les accusés qui ont commis seul leur crime et qui n'ont pas agi sous influence, la défense ne change guère: on met en avant l'irresponsabilité naturelle de l'enfant. Rappelons en effet que si le crime est commis avant la seizième année de l'accusé, la question du discernement est systématiquement posée en vertu des articles 66 et $67 \mathrm{du}$ code pénal. Dès lors, la tâche de l'avocat de la défense est au mieux de faire déclarer son client non coupable, au pire d'obtenir une réponse négative à la question du discernement. Une tâche simplifiée par la volonté des magistrats d'appliquer le plus souvent possible l'article 66 du code pénal. Dans la rubrique judiciaire, l'association de la minorité pénale à l'absence de discernement est quasi systématique : Marie Vincent est « coupable d'avoir dérobé du blé à ses maîtres à une époque où elle n'avait pas seize ans révolus, et ainsi d'avoir agi sans discernement», lit-on dans l'Ami de la Patrie. ${ }^{73} \mathrm{La}$ grande majorité des actes d'accusations étudiés dans le cadre de notre réflexion insiste toutefois sur une responsabilité totale, malgré leur âge, des mineurs accusés : François Aussize « est dans sa douzième année, mais il n'est pas nécessaire que le discernement soit bien développé pour comprendre qu'en portant si violemment des coups répétés, sur la tête d'une personne, à l'aide d'un instrument de fer, on s'expose à la lui briser ". ${ }^{74}$ La mesure du discernement d'un mineur fait rarement l'objet d'un suivi des experts : on laisse à la discrétion de la cour d'assises, et au talent de l'avocat, le soin d'établir le degré de responsabilité de l'accusé. Quelques exceptions cependant: dans l'affaire de 
Cournon en 1871, le docteur Welter indique avec « une parfaite mesure le degré de responsabilité qui incombe, d'après lui, à chacun des accusés $",{ }^{75}$ dans l'affaire Joseph Prat en 1885, « il résulte du rapport de l'homme de l'art qui l'a observé ou fait observer pendant près d'un mois et demi, que le jeune Prat n'a durant cette période donné aucun signe de crises épileptiformes, qu'il a toujours répondu avec clarté et précision aux questions qui lui ont été posées et qu'il est responsable de ses actes autant que peut l'être un enfant de douze ans $»{ }^{76}$ La question du discernement d'un point de vue strictement pénal, apparaît donc aux yeux de la chronique comme une formalité que l'on n'associe absolument pas avec le réel degré de conscience de l'enfant ou de l'adolescent: «Une [des] deux dernières questions concerne notamment le jeune Chauvon, Jules, qui n'avait pas seize ans accomplis au moment du crime et qui a, en conséquence, agi sans discernement $» .^{77}$

\section{Corriger le jeune criminel}

13 Sur les 60 accusés retenus pour cette étude, 26 ont commis leur crime dans leur seizième année (ou leur dix-huitième année à partir d'août 1906) et sont donc jugés comme des adultes. 5 d'entre eux sont condamnés à des peines de travaux forcés, 12 à des peines de prison et 9 sont déclarés non coupables et remis en liberté. Excepté pour Antoine Vray, tous les accusés reconnus coupables bénéficient soit de circonstances atténuantes, soit de réponses négatives aux questions aggravantes. Si l'on s'intéresse plus spécifiquement aux 34 accusés âgés de moins de 16 ans (moins de 18 ans à partir d'août 1906) au moment des faits, 21 sont reconnus coupable dont 14 comme ayant agi sans discernement et 11 sont acquittés et remis en liberté. ${ }^{78}$ L'étude des réquisitoires laisse entrevoir un premier degré d'indulgence, plutôt inattendu, de la part du ministère public à l'égard de la plupart de ces accusés. «En ce qui touche Bourguignon, M. l'avocat général laisse aux jurés le soin d'estimer si, oui ou non, cet accusé, presqu'enfant, doit obtenir un peu d'indulgence ", lit-on dans le Moniteur du Puy-deDôme. ${ }^{79}$ C'est encore le ministère public qui demande que l'on accorde au jeune Binet et à son complice Convert, "en raison de leur jeune âge, le bénéfice des circonstances atténuantes $» .^{80}$ Cette indulgence du ministère public ne doit toutefois pas cacher la réalité de la répression pénale à l'égard de ces jeunes mineurs. L'issue d'un procès ne varie guère : que l'on reconnaisse ou non l'absence de discernement et que l'on accorde ou non les circonstances atténuantes, ils seront pour la plupart envoyés en prison ou en maison de correction, ce qui amène l'avocat du jeune Achille Vaisse à poser la question suivante : «A-t-il agi avec discernement? Vaisse a quinze ans à peine. Quel sera le résultat du verdict de messieurs les jurés? Que ce verdict soit affirmatif ou négatif le résultat sera le même, la loi laissant dans ce dernier cas à la cour la faculté d'envoyer l'enfant dans une maison de correction. » Une ambiguïté qui, déjà en 1857, faisait réagir le conseiller Burin-Desroziers lors du procès du jeune André Mondière: "La peine qu'on peut leur infliger en cas de condamnation n'est pas la peine ordinaire; on ne peut les condamner qu'à rester dans une maison de correction pendant un temps dont la durée varie entre dix et vingt ans» ${ }^{81}$ L'enfermement en maison de correction apparaît dans le discours retranscrit de la justice comme la seule et unique solution pour remettre ces enfants sur le droit chemin. Toujours dans l'affaire des incendies commis par le jeune Mondière, l'avocat général Ancelot demande l'envoi de l'enfant en maison de correction, "où peut-être les bons exemples qu'il aura sous les yeux, finiront par le corriger ${ }^{82}$ Le président de la session, dans son résumé, suit les 
recommandations du ministère public, il « fait remarquer à quel point il est utile d'user de tous les moyens possibles pour corriger un enfant qui n'a pas craint de dire à l'épouse du brigadier de gendarmerie de Chabreloche : «Quand tout le village de SaintJoany aurait été brûlé, j'aurais été mettre le feu dans les communes voisines ${ }^{83}$ Le renvoi de l'enfant auprès de sa famille est une alternative rarement envisagée. Sur les 18 accusés de moins de 16 ans reconnus coupables, 17 sont envoyés en maison de correction et seul Jean Batisse, 15 ans, est remis à ses parents. La défaillance de l'éducation donnée à l'enfant, la précarité des conditions de vie des parents, voire l'absence totale de famille, constituent pour la justice un risque inévitable de récidive que l'on doit à tout prix éviter : «Enlever des jeunes à des familles défaillantes, tant du point de vue social que moral, les confier à l'Etat pour qu'il offre une éducation appropriée, même si c'était dans un cadre carcéral, explique l'usage de plus en plus fréquent de l'article 66 afin de préserver et de moraliser de jeunes victimes. »

Pour les avocats de la défense, l'équation est simple : envoyer un mineur en maison de correction, c'est ne lui offrir aucun avenir : "Va-t-on briser ces existences qui peuvent être encore utiles à la société ? » s'exclame le défenseur de Queyriaux, qui propose une autre solution pour son jeune client : "Qu'on leur permette d'aller sous les drapeaux, effacer leur faute en protégeant la patrie. $\aleph^{84}$ Antichambre d'un milieu carcéral corrompant, il apparaît très clairement, dans le discours de la défense, que la maison de correction ne corrige en rien celui qu'on y envoie. A partir des années 1890, l'argument devient un classique des plaidoiries : Journet, le défenseur du jeune Montel, supplie le jury le 28 novembre 1893 « de ne pas permettre qu'on envoie [son client], qui se repent d'un enfantillage, dans une maison de correction où il ne puiserait que de mauvais principes et d'où il sortirait malfaiteur dangereux et incorrigible ». ${ }^{85} \mathrm{Il}$ dresse alors « un sombre tableau de la promiscuité dangereuse régnant dans les maisons de correction d'où rarement on sort avec de bons instincts $»{ }^{86}$ Le concept de la prison école du crime est omniprésent dans les articles de presse de la fin du XIXe siècle, nous rappelle Christophe Israël dans son étude des jeunes insoumis de quartier correctionnel, et citant le rédacteur du Journal de Rouen en 1892 : «Il est reconnu depuis longtemps que la maison de correction est une mauvaise école et que, dans la plupart des cas, l'enfant achève d'y perdre par la fréquentation de camarades encore plus corrompus que lui le peu de bons sentiments qui pouvaient lui rester $\$^{87}$. Pourtant, si quelques morceaux choisis de plaidoiries laissent entrevoir, dans la chronique judiciaire puydomoise, une critique des conditions d'enfermement de jeunes détenus, on ne s'étend pas sur le sujet. Difficile, voire impossible, à partir de ces comptes rendus d'audience, de dresser un tableau du système carcéral départemental et d'observer la dangereuse promiscuité que le conseiller Lacarrière de la cour d'appel de Riom dénonçait encore le 27 janvier 1873 dans l'enquête parlementaire sur le régime des établissements pénitentiaires.$^{88}$ L'itinéraire des jeunes accusés des maisons d'arrêts départementales à la Maison Centrale de Riom, leurs conditions de détention et leur périple au sein des colonies pénitentiaires sont autant de thèmes qui ne trouvent aucun écho dans les colonnes des rubriques judiciaires locales ${ }^{89}$ Les portes de la maison de correction, raccourci idéal, un de plus, pour désigner l'ensemble des institutions pénitentiaires de jeunes détenus, restent définitivement closes au regard du lectorat. Par ailleurs, on se garde bien de donner un avis sur les verdicts et les peines prononcées. L'engagement politique de chaque journal, celui-là même qui anime les polémiques et alimente les débats de l'actualité nationale de la première page ne semble pas être en mesure de troubler la litanie codifiée de la rubrique judiciaire. 


\section{Conclusion}

Parce qu'ils ne font que suivre des individus plus âgés, leurs frères ainés ou leurs parents, une grande majorité des mineurs jugés par la cour d'assises du Puy-de-Dôme sont les dignes représentants d'une criminalité d'accompagnement vouée à rester dans l'ombre des projecteurs médiatiques. Sur les 176 comptes rendus d'audience que nous avons étudiés (tous journaux confondus), plus de $78 \%$ ne couvrent pas plus qu'un quart d'une page et rares sont les affaires qui bénéficient de plus d'un article par journal consacré à l'enquête. Trois figures stéréotypées de jeunes criminels profitent toutefois de l'essor et du développement de la presse dès les premières années de la Troisième République: le jeune meurtrier, l'agresseur nocturne et l'enfant incendiaire. Si le Moniteur du Puy-de-Dôme n'accorde que quelques lignes au coup de couteau d'Auguste Robillon en 1858, le crime de sang s'affiche davantage avec l'affaire Ventalon en 1871 pour atteindre son apogée au début du XXe siècle avec les crimes des familles Touche et Roussel, les seules affaires qui auront le privilège des gros titres et d'un suivi quotidien de l'instruction par la plupart des journaux. Dans les années 1890-1900, les affaires d'agressions nocturnes commises par des mineurs doivent leur éclairage médiatique à leur assimilation aux grands thèmes sécuritaires de la fin du XIXe siècle: l'Apache provincial est né. Quant aux répercussions médiatiques des incendies volontaires commis par des enfants, crimes à la fois graves, exceptionnels et intemporels, elles sont à l'image de l'effroi que peuvent susciter ces actes de destruction en milieu rural..$^{90}$ Des répercussions qui toutefois n'évoluent guère au fil des décennies qui séparent les premières années du Second Empire de la Belle Epoque. Les vols domestiques et les crimes sexuels, faute de présenter les conditions nécessaires pour s'étendre dans les rubriques criminelles, sont voués à ne produire qu'une actualité marginale, celle des " nouvelles à trois lignes ". ${ }^{91}$ L'intérêt que suscitent les affaires mettant en cause des accusés mineurs reste donc largement tributaire de la nature et de la gravité du ou des crimes commis. L'absence de polémiques et de réactions politiques à l'égard de la déviance des plus jeunes peut paraître plus surprenante au premier abord, surtout à partir des années 1880, alors que les débats idéologiques accaparent l'actualité nationale et locale des principales feuilles du département. Point de discours alarmistes sur le déclin des mœurs ni de condamnations d'un système corrompu et corrupteur, les bases de la société ne semblent pas fragilisées par les exactions commises par ces jeunes désintégrés. Vivant au sein d'une population en marge de la société, ni représentants ni reflets des jeunesses rurales et urbaines normalisées, ils n'existent que par leur déviance régulière ou passagère. La paresse, la débauche, l'alcool, le vagabondage, la misère sociale et la perversité naturelle sont les facteurs criminogènes que l'on expose le plus dans les colonnes de la chronique judiciaire, quelle que soit la période étudiée. Reste que, face à l'absence d'un débat, on évoque plus que l'on stigmatise, on rappelle plus que l'on interpelle. Finalement, on divertit plus que l'on questionne. Cette pauvreté de l'engagement politique, idéologique et intellectuel, nous la retrouvons enfin dans l'évocation des solutions judiciaires à apporter à la criminalité des plus jeunes. Une fois de plus, rien ne se discute : la question du discernement, les peines encourues, le problème de la correction et de la réinsertion, on se contente de reprendre, ponctuellement et partiellement, quelques réquisitoires et plaidoiries bien menés sans aucune autre volonté que d'en féliciter l'orateur. 


\section{NOTES}

1. Le nombre de mineurs jugés par la cour d'assises du Puy-de-Dôme reste stable jusque dans les premières années du XXe siècle : 1,6\% du nombre total d'accusés sur la période 1852-1871 et 1872-1891. L'intégration des accusés de 17 et 18 ans à partir d'avril 1906 fait sensiblement augmenter ce taux sur la période 1892-1912:2,3\%.

2. L'article 68 du code pénal stipule que « l'individu, âgé de moins de seize ans, qui n'aura pas de complices présents au-dessus de cet âge, et qui sera prévenu de crimes autres que ceux que la loi punit de la peine de mort, de celle des travaux forcés à perpétuité, de la peine de la déportation ou de celle de la détention, sera jugé par les tribunaux correctionnels ».

3. Les coups et blessures qualifiés et les abus de confiance/faux et usage de faux concernent $5 \%$ des accusés chacun et les vols domestiques $3,3 \%$. Ces chiffres suivent d'assez près ceux du Compte Général de l'administration de la justice criminelle de 1852 à 1892 sur le plan national.

4. Sur les Apaches : Michelle Perrot, Les ombres de l'histoire, crime et châtiment au XIXe siècle, Paris, Flammarion, 2001.

5. Sylvie Lapalus, «La famille assiégée de l'intérieur : jeunes parricides au XIX ${ }^{\text {ème }}$ siècle», Revue d'histoire de l'enfance irrégulière, Numéro 3, 2000, http://rhei.revues.org/document50.html.

6. Sans titre, Moniteur du Puy-de-Dôme, 22/07/1876, p.3.

7. Jean-Claude Farcy, La jeunesse rurale dans la France du XIXe siècle, Paris, Editions Christian, 2004, p.113.

8. «Affaire de Cournon. - Meurtre. 8 accusés ", Gazette d'Auvergne, 14/02/1874, p.3.

9. Ibid.

10. " Audience du 26 novembre », Presse Judiciaire, 03/12/1855, p.2.

11. «Incendies ", Moniteur du Puy-de-Dôme, 17/02/1857, pp.2-3.

12. «Incendie volontaire », Moniteur du Puy-de-Dôme, 07/03/1885, p.3

13. "Audience du 13 mai », Ami de la Patrie, 15/05/1853, p.3.

14. "Le crime de Sayat ", Moniteur du Puy-de-Dôme, 09/08/1907, p.2.

15. «Assassinat ", Indépendant du Centre, 01/06/1871, p.3.

16. «Vol qualifié et complicité », Moniteur du Puy-de-Dôme, 12/05/1887, p.3.

17. "Audience du 28 février", Ami de la Patrie, 03/03/1853, p.2.

18. "Suite de l'audience du lundi 28 février 1853 », Journal du Puy-de-Dôme, 03/03/1853, p.2.

19. " Audience du 26 novembre », Presse Judiciaire, 03/12/1855, p.2.

20. «Affaire Saint-Cyr-Bourguignon. Vol qualifié », Petit Clermontois, 16/11/1892, p.2.

21. "Vol qualifié et complicité », Riom Journal, 04/03/1894, p.2.

22. Sylvie Lapalus, op. cit.

23. «Incendie », Le Moniteur du Puy-de-Dôme, 19/11/1859, p.3.

24. Jean-Claude Vimont, «Des coupables aux victimes, l'archéologie de l'identité du mineur délinquant au XIXème siècle ", in Jean-Claude Vimont (dir.), "Jeunes, déviances et identités, XVIIIe-XXe siècle", Cahiers du GRHIS, n 15 , Rouen, Publications des Universités de Rouen et du Havre, 2005, p.35.

25. "Suite de l'audience du 21 », Journal du Puy-de-Dôme, 24/08/1854, p.2.

26. "Affaire de Cournon. Meurtre, 8 accusés ", Gazette d'Auvergne, 14/02/1874, p.3.

27. "Audience du 28 février", Ami de la Patrie, 03/03/1853, p.2.

28. Nous emploierons ce terme plutôt que la notion d'absence de discernement pour éviter la confusion avec la définition pénale de ces termes.

29. "L'agression de Manzat ", Petit Clermontois, 26/05/1893, p.3.

30. «Le vol de la rue Neuve ", Petit Clermontois, 09/08/1890, p.2.

31. "Affaire Chassagnon. Vol qualifié », Petit Clermontois, 03/02/1892, p.2. 
32. «Assassinat », Indépendant du Centre, 01/06/1871, p.3.

33. "Assassinat suivi de vol commis par un garçon de 16 ans ", Riom Journal, 29-30/05/1871, pp. 3-4.

34. "Le meurtre de Sayat ", Avenir du Puy-de-Dôme, 09/08/1907, p.2.

35. "Le crime de Sayat ", Moniteur du Puy-de-Dôme, 09/08/1907, p.2.

36. Le pourcentage est certainement plus important dans la mesure où dans $47 \%$ des affaires étudiées, les informations sur la condition sociale de la famille ne sont pas suffisamment précises pour permettre de les intégrer dans la catégorie des familles précaires.

37. «Vols qualifiés ", Moniteur du Puy-de-Dôme, 04/08/1859, p.3.

38. «Incendie volontaire », Moniteur du Puy-de-Dôme, 07/03/1885, p.3.

39. "Audience du 13 mai », Journal du Puy-de-Dôme, 15//05/1853, p.2.

40. «Vols qualifiés", Moniteur du Puy-de-Dôme, 21/08/1880, p.3.

41. "Vol qualifié et complicité ", Moniteur du Puy-de-Dôme, 12/05/1887, p.3.

42. "L'assassinat de Culhat ", Le Moniteur du Puy-de-Dôme, 07/08/1903, p.2.

43. "Le crime de Sayat ", Moniteur du Puy-de-Dôme, 09/08/1907, p.2.

44. Ibid.

45. «Vol qualifié », Indépendant du Centre, 16/11/1871, p.4.

46. "Vol qualifié », Moniteur du Puy-de-Dôme, 09/08/1890, pp.2-3.

47. "Affaire Chassagnon. Vol qualifié », Petit Clermontois, 03/02/1892, p.2.

48. "Vols qualifiés", Moniteur du Puy-de-Dôme, 16/05/1883, p.3.

49. "Audience du 26 novembre », Presse Judiciaire, 03/12/1855, p.2.

50. «Audience du 3 », Riom Journal, 05/03/1871, p.3.

51. «Vols", Moniteur du Puy-de-Dôme, 13/08/1865, p.2.

52. Jean-Jacques Yvorel, «De Gavroche aux apaches. Source et méthode d'une histoire des illégalismes juvéniles ", in Frédéric Chauvaud, Jacques-Guy Petit (dir.), L'histoire contemporaine et les usages des archives judiciaires (1800-1939), Histoire et archives, hors série $\mathrm{n}^{\circ} 2$, Paris, Honoré Champion Editeur, 1998, p.453 et «Vagabondage des mineurs et politique pénale en France de la Restauration à la République des Ducs » in Jean-Claude Caron, Annie Stora-Lamarre, Jean-Jacques Yvorel (dir.), Les Ames mal nées. Jeunesse et délinquance urbaine en France et en Europe, XIXe-XXIe siècles, Besançon, Presses universitaires de Franche-Comté, 2008, p.63.

53. "Affaire Aimard et autres. Vols qualifiés ", Moniteur du Puy-de-Dôme, 14/08/1875, pp.2-3.

54. «Incendie volontaire ", Moniteur du Puy-de-Dôme, 07/03/1885, p.3.

55. « Vol ", Ami de la Patrie, 29/11/1855, p.3.

56. Acte d'accusation, affaire Paul/Aimard/Denis, dossier 6191, série U 10824, 1875.

57. «L'affaire de Manzat. Agression et vol », Moniteur du Puy-de-Dôme, 06/04/1893, p.2.

58. "Même audience ", Indépendant du Centre, 25/05/1871, p.3.

59. «Le vol de la rue Neuve ", Petit Clermontois, 09/08/1890, p.2.

60. «Incendies ", Moniteur du Puy-de-Dôme, 17/02/1857, pp.2-3.

61. «Vols qualifiés ", Riom Journal, 17/05/1883, p.3.

62. Sylvie Lapalus, op. cit.

63. «Infanticide, Gazette d'Auvergne, 25/08/1876, p.3.

64. "L'assassinat de Culhat», L'Avenir du Puy-de-Dôme, 07/08/1903, p.2-3.

65. Jean-Claude Vimont, «Des coupables aux victimes, l'archéologie de l'identité du mineur délinquant au XIXème siècle ", dans Jean-Claude Vimont (dir.), "Jeunes, déviances et identités, XVIIIe-XXe siècle", Cahier du GRHIS, n¹5, Rouen, Publications des Universités de Rouen et du Havre, 2005, p.44.

66. "Audience du 13 mai », Ami de la Patrie, 15/05/1853, p.3

67. «Vol. Trois accusés ", Ami de la Patrie, 15/11/1854, p.2

68. "Affaire Jouberton, Retrus et Vaisse. Vols qualifiés ", Moniteur du Puy-de-Dôme, 04/12/1875, pp.3-4. 
69. «Vol qualifié », Moniteur du Puy-de-Dôme, 25/02/1880, p.3.

70. «Affaire Saint-Cyr-Bourguignon. Vol qualifié », Petit Clermontois, 16/11/1892, p.2.

71. "Affaire Chassagnon ", Petit Clermontois, 04/02/1892, p.2.

72. « Le vol de la rue Neuve », Petit Clermontois, 09/08/1890, p.2.

73. «Vol. Trois accusés ", Ami de la Patrie, 15/11/1854, p.2.

74. Acte d'accusation, affaire Aussize, dossier 6011, série U 10812, 1871.

75. « Affaire de Cournon. Meurtre. 8 accusés », Gazette d'Auvergne, 14/02/1874, p.3.

76. Acte d'accusation, affaire Prat, dossier 6583, série U 10859, 1885.

77. «Vol qualifié et complicité ", Moniteur du Puy-de-Dôme, 12/05/1887, p.3.

78. Deux cas particuliers : Joseph Frugère est condamné par contumace le 6 mars 1885 à 20 ans de travaux forcés. Il sera retrouvé et condamné deux ans plus tard à un an de maison de correction. Quant à Antoine Champommier, après avoir été acquitté du crime de viol, l'avocat général demanda à ce qu'il fut retenu en prison pour attentat à la pudeur. La cour a fait droit au réquisitoire du ministère public et réintégra le jeune acquitté dans la maison d'arrêt. Nous n'avons trouvé aucune trace de son jugement pour attentat à la pudeur.

79. "Audience de l'après-midi », Moniteur du Puy-de-Dôme, 16/11/1892, pp.2-3.

80. "L'agression de Manzat. Vol qualifié », Moniteur du Puy-de-Dôme, 26/05/1893, p.2.

81. "Incendies ", Moniteur du Puy-de-Dôme, 17/02/1857, pp.2-3.

82. "Incendies », Journal du Puy-de-Dôme, 16-17/02/1857, pp.2-3.

83. Ibid.

84. "Affaire Aimard et autres. Vols qualifiés », Moniteur du Puy-de-Dôme, 14/08/1875, pp.2-3.

85. "Incendie volontaire ", Moniteur du Puy-de-Dôme, 29/11/1893, pp.2-3.

86. «Incendie volontaire ", Riom Journal, 30/11/1893, p.2.

87. Christophe Israël, « Le profil des jeunes insoumis d'un quartier correctionnel à la fin du XIXe siècle ", dans Jean-Claude Vimont (dir.), "Jeunes, déviances et identités, XVIIIe-XXe siècle", Cahiers du GRHIS, n¹5, Rouen, Publications des Universités de Rouen et du Havre, 2005, p.75.

88. Eric Pierre, Marie-Sylvie Dupont-Bouchat (dir.), Enfance et Justice au XIXe siècle, Paris, PUF, 2001, p.133. L'enquête parlementaire sur le régime des établissements pénitentiaires de 1872-1875 peut être consultée en ligne sur le site de l'ENAP, dans la rubrique Ressources en lignes.

89. L'histoire de la maison centrale de Riom sous la Troisième République a fait l'objet d'un mémoire de maîtrise : Marion Couturier, La maison centrale de Riom sous la troisième République : 1871-1914, Clermont-Ferrand, Université Blaise Pascal, 2004.

90. Jean-Claude Caron, «Figures du jeune incendiaire dans la France du XIXe siècle, entre scène judiciaire et regard politique» dans Jean-Claude Caron, Annie Stora-Lamarre, Jean-Jacques Yvorel (dir.), Les Ames mal nées. Jeunesse et délinquance urbaine en France et en Europe, XIXe-XXIe siècles, Besançon, Presses universitaires de Franche-Comté, 2008, p.49.

91. Dominique Kalifa, Crime et culture au XIXe siècle, Paris, Perrin, 2005, p.319.

\section{INDEX}

Index géographique : France, Puy-de-Dôme

Mots-clés : Cour d'assise, exposition médiatique, média, presse

Index chronologique : Milieu du XIXème siècle, Fin du XIXème siècle, Début du XXème siècle 
AUTEUR

SÉBASTIEN SOULIER

Doctorant en histoire, université de Clermont-Ferrand 